\title{
CD-Monitored HTS of Chiral Catalysts
}

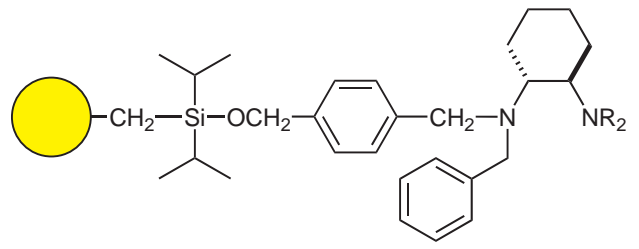

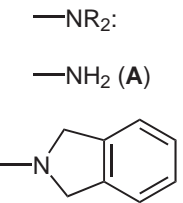

(B)<smiles>CN1Cc2cccc(Cl)c2-c2c(ccc3ccccc23)C1</smiles><smiles>CN1Cc2ccc3ccccc3c2-c2c1ccc1ccccc21</smiles>

$[\mathrm{A}, \mathrm{B}, \mathrm{C}, \mathrm{D}] \times\left[\mathrm{CuCl}(1), \mathrm{CuCl}_{2}(2), \mathrm{Cu}(\mathrm{OAC})_{2}(3)\right]=$ a library of polymeric catalysts A1-D3 (12 members)

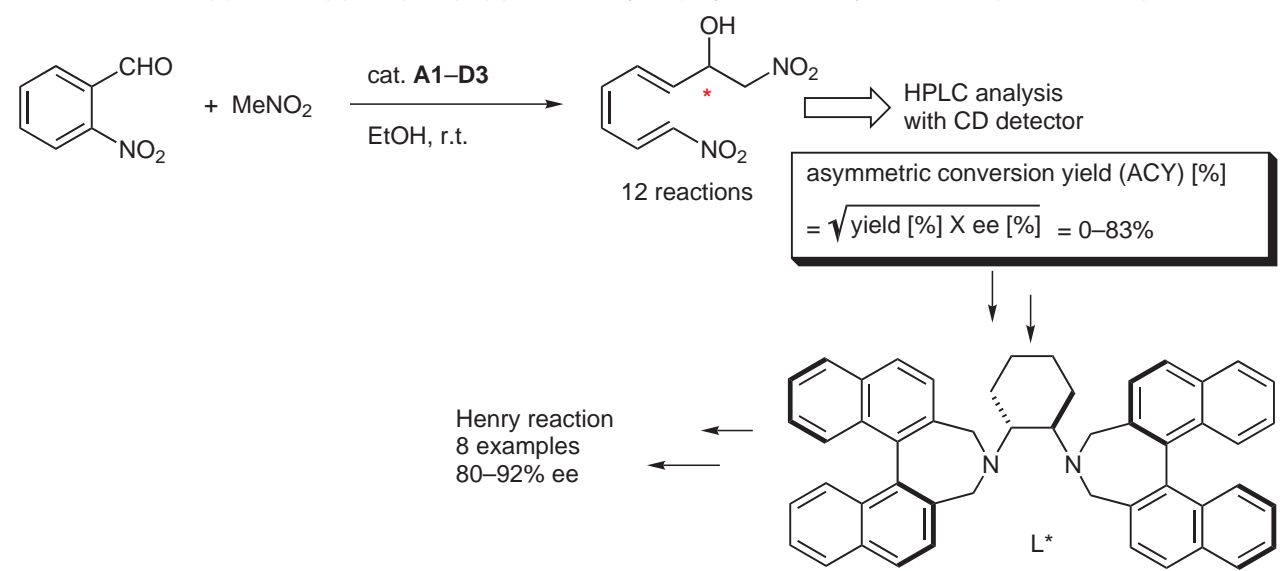

Key words

asymmetric catalysis

circular dichroism

combinatorial chemistry

high-throughput screening

Significance: High-throughput screening (HTS) of chiral catalysts was performed with flow-CD detector. A combinatorial library of chiral diaminecopper catalysts (12 members) was prepared on polystyrene support (beads). Catalytic efficiency was examined for the Henry reaction of 2-nitrobenzaldehyde with nitromethane and evaluated by the asymmetric conversion yield (ACY) value. Signal intensity obtained via analysis using the CD detector provided the ACY value in which the information of both asymmetric induction ability and catalytic activity were combined in one signal data. Catalyst D1 was identified to be an effective lead catalyst for the asymmetric Henry reaction. Through the lead optimization, $\mathrm{L}^{*}-\mathrm{CuCl}$ was found to promote the Henry reaction with high enantioselectivity and high catalytic activity (8 examples with 6 aldehydes).
Comment: Utility of the HPLC-CD system for the HTS of chiral catalysts has already been reported independently by the group of Mikami and Ding and that of Reetz. The pioneering reports required chromatographical purification (or separation) of the target molecule because each of the test reactions was carried out with a unique homogeneous catalyst. The present protocol was performed with a solid-supported catalyst library to simplify the post treatment of ACY evaluation. 